\title{
Perfil sorológico dos anticorpos colostrais para Neospora caninum em bezerros livres da infecção
}

José Márcio S. CARDOSO'1 Mikaela Renata FUNADA ${ }^{1}$ Rodrigo Martins SOARES ${ }^{1}$ Solange Maria GENNARI ${ }^{1}$

Correspondência para:

Av. Prof. Orlando Marques de Paiva, 87, Cidade Universitária, 05508-000, São Paulo-SP; sgennari@usp.br

Recebido para publicação: 04/12/2007 Aprovado para publicação: 29/05/2008

1 - Departamento de Medicina Veterinária Preventiva e Saúde Animal da Faculdade de Medicina Veterinária e Zootecnia da Universidade de São Paulo, São Paulo-SP

\section{Resumo}

Neospora caninum é considerado a principal causa de aborto bovino mundial. O diagnóstico laboratorial correto é muito importante para identificar os animais infectados e para aplicar medidas de controle. $\mathrm{O}$ objetivo deste trabalho foi mostrar o declínio de anticorpos colostral em bezerros. Este estudo empregou oito bezerros holandeses, recémnascidos, machos, descendentes de vacas soronegativas para $N$ caninum. Amostra de sangue pré-colostral foram colhidas destes bezerros e todos estavam soronegativos pra $N$. caninum. Estes bezerros foram alimentados com dois litros de um pool de colostro de vacas soropositivas dentro de duas horas após o nascimento. Amostras de sangue dos bezerros foram colhidas semanalmente até os animais soroconverterem negativo. As amostras foram testadas para anticorpos de $N$. caninum usando teste de imunofluorescência indireta nos títulos de 1:50; 1: 100 e 1:200. Os resultados mostraram que 3 dos 8 bezerros não soroconverteram e foram excluídos do estudo. Os restantes cinco bezerros soroconverteram em todos os títulos no quinto dia após a inoculação. No título 1:50, um bezerro permaneceu positivo por 21 semanas, dois por 20 semanas e um por 13 semanas. No título 1:100, um bezerro foi positivo por 15 semanas e o restante quatro bezerros por 13 semanas. No título 1:200, cada bezerro foi positivo por 1; 7; 12; 12 e 13 semanas, respectivamente. Estes resultados demonstram que o anticorpo colostral para $N$. caninum pode permanecer até 21 semanas após o nascimento nos bezerros e é muito importante excluir os bezerros até quatro meses de idade nos estudos de soroprevalência para impedir os resultados falso-positivos.

\section{Introdução}

Deste seu primeiro reconhecimento em 1984 em cães na Noruega ${ }^{1}$ e a descrição de um novo gênero e espécie Neospora caninum $^{2}$, este coccídeo tem emergido como um sério problema mundial em cães e bovinos $^{3}$. Estudos demonstraram que cães $^{4,5,6}$ e coiotes ${ }^{7}$ são hospedeiros definitivos para $N$. caninum.

Em bovinos, transmissão vertical ou congênita do $N$. caninum é considerada a via de transmissão mais importante, onde até 95\% dos bezerros nascidos de vacas soropositivas foram infectados congenitamente e clinicamente normais. $8,9,10,11,12,13,14,15,16,17,18,19,20,21,22$

Estudos sorológicos têm demonstrado evidências de transmissão horizontal através da falta de associação entre o status sorológico dos pares mães-filhas, da diferença da soroprevalência entre grupos de diferentes faixas etárias ou de lotes de separação; soroconversões; respostas sorológicas transitórias; curva epidêmica durante um surto de aborto associado ao $N$. caninum e infecção aguda baseada no ELISA

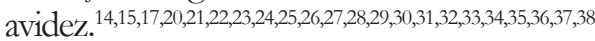

Existe evidência em camundongos sugerindo que a transmissão lactogênica de N. caninum pode ocorrer, como demonstrado por Cole et $a .^{39}$ em um dos 
51 camundongos lactentes de uma fêmea infectada experimentalmente. Transmissão lactogênica de $N$. caninum foi demonstrada experimentalmente em bezerros recémnascidos alimentados com colostro ou leite inoculados com taquizoítas ${ }^{40,41}$, mas, não há evidências, de que ocorra transmissão em infecções naturais ${ }^{41}$.

A alimentação de bovinos recémnascidos com pool de colostro e leite é uma prática bastante utilizada em propriedades leiteiras em algumas regiões do Brasil e do mundo e o conhecimento do perfil dos anticorpos contra $N$. caninum em bezerros leiteiros é de grande importância para a interpretação de dados sorológicos obtidos em bovinos jovens. Assim o objetivo deste estudo foi determinar, em neonatos bovinos soronegativos para N. caninum, o perfil dos anticorpos anti- $N$. caninum adquiridos passivamente pelo colostro.

\section{Material e Método}

O estudo foi conduzido no Campus Administrativo da Universidade de São Paulo, no município de Pirassununga, SP. Foram empregados oito bezerros holandeses, recém-nascidos, machos, filhos de vacas soronegativas para $N$. caninum pela reação de imunofluorescência indireta (RIFI) com título $\leq 25$. As vacas doadoras de colostro (cinco animais) eram soropositivas para N. caninum (RIFI >100). O colostro desses animais foi colhido, identificado, acondicionado em frascos apropriados e armazenado à $-20^{\circ} \mathrm{C}$ até a sua utilização.

Os bezerros recém nascidos não foram alimentados com leite ou colostro materno ou de outro animal, recebendo, em até 2 horas após o nascimento, oralmente, através de mamadeiras, dois litros do colostro previamente obtido para este estudo.

Antes da administração do colostro, uma amostra de sangue foi colhida para confirmar que anticorpos anti- $N$. caninum não estavam presentes. Estes animais foram mantidos em baias coletivas com quatro bezerros por baia e receberam leite em pó comercial até o desmame, aos 60 dias de idade. Ração comercial e feno foram fornecidos em quantidades adequadas à idade e água ad libitum. Estes procedimentos tiveram por finalidade impedir possíveis infecções pelo $N$. caninum após o nascimento.

Amostras de sangue foram colhidas um dia após a ingestão do colostro e depois uma vez por semana de todos os animais para acompanhamento dos níveis de anticorpos séricos anti- $N$. caninum. A técnica utilizada foi a RIFI segundo metodologia descrita por Dubey et al. ${ }^{42}$. Todas as amostras foram processadas em três diluições: 1:50, 1:100 e 1:200 e soros bovinos controles positivos e negativos foram processados. Amostras de sangue foram colhidas até que todos os animais apresentassem resultados negativos, em todas as diluições, por três semanas consecutivas, quando os animais foram considerados negativos.

\section{Resultados e Discussão}

Todas as cinco vacas doadoras de colostro apresentaram anticorpos anti- $N$. caninum com título de 1:100. Os bezerros usados no estudo e suas respectivas mães estavam negativas pela RIFI com título $\leq 25$, quando do nascimento.

A tabela 1 apresenta os resultados da sorologia dos bezerros durante as 24 semanas de observação. Os bezerros número 2, 3 e 4 receberam colostro da vaca 5729; os bezerros 1 e 5 das vacas 3287 e 5807 , respectivamente. Três bezerros não soroconverteram, dos quais dois haviam sido alimentados com colostro da mesma vaca (4060) e o outro com colostro da vaca 3118. Estes três bezerros que não soroconverteram nas quatro primeiras amostragens foram retirados do estudo.

Após sete dias da administração do colostro cinco bezerros tinham anticorpos anti-N. caninum pela RIFI nas três diluições estudadas. Com exceção do bezerro número 5, que se tornou negativo da diluição 1:200 na segunda semana pós-colostro, os outros bezerros apresentavam títulos nas três diluições até a sétima semana. Algumas 
Tabela 1 - Resultados da Reação de Imunofluorescência Indireta, (P - Positivo; N - negativo) nas diluições de $1: 50,1: 100$ e 1:200, dos soros de bezerros negativos e alimentados com colostro de vacas positivas para Neospora caninum, nas primeiras 24 semanas de vida

\begin{tabular}{|c|c|c|c|c|c|c|c|c|c|c|c|c|c|c|c|}
\hline \multirow{3}{*}{ SEMANA } & \multicolumn{15}{|c|}{ NÚMERO DOS BEZERROS } \\
\hline & \multicolumn{4}{|c|}{1} & \multicolumn{2}{|c|}{2} & \multicolumn{3}{|c|}{3} & \multicolumn{3}{|c|}{4} & \multicolumn{3}{|c|}{5} \\
\hline & $1: 50$ & $1: 100$ & $1: 200$ & $1: 50$ & $1: 100$ & $1: 200$ & $1: 50$ & $1: 100$ & $1: 200$ & $1: 50$ & $1: 100$ & $1: 200$ & $1: 50$ & $1: 100$ & $1: 200$ \\
\hline 1 & $\mathrm{P}$ & $\mathrm{P}$ & $\mathrm{P}$ & $P$ & $\mathrm{P}$ & $\mathrm{P}$ & $P$ & $\mathrm{P}$ & $\mathrm{P}$ & $\mathrm{P}$ & $\mathrm{P}$ & $\mathrm{P}$ & $P$ & $\mathrm{P}$ & $P$ \\
\hline 2 & $\mathrm{P}$ & $\mathbf{P}$ & $\mathrm{P}$ & $\mathbf{P}$ & $P$ & $\mathbf{P}$ & $\mathbf{P}$ & $\mathbf{P}$ & $P$ & $\mathbf{P}$ & $\mathbf{P}$ & $P$ & $\mathbf{P}$ & $\mathrm{P}$ & $\mathrm{N}$ \\
\hline 3 & $\mathrm{P}$ & $\mathrm{P}$ & $\mathrm{P}$ & $\mathrm{P}$ & $\mathrm{P}$ & $\mathrm{P}$ & $\mathrm{P}$ & $\mathrm{P}$ & $\mathrm{P}$ & $\mathrm{P}$ & $\mathbf{P}$ & $P$ & $\mathrm{P}$ & $\mathrm{P}$ & $\mathrm{N}$ \\
\hline 4 & $\mathrm{P}$ & $\mathrm{P}$ & $\mathrm{N}$ & $P$ & $\mathrm{P}$ & $\mathrm{P}$ & $P$ & $\mathrm{P}$ & $P$ & $\mathrm{P}$ & $\mathrm{P}$ & $P$ & $\mathrm{P}$ & $\mathrm{P}$ & $\mathrm{N}$ \\
\hline 5 & $\mathrm{P}$ & $\mathrm{P}$ & $\mathrm{N}$ & $\mathrm{P}$ & $\mathrm{P}$ & $\mathrm{P}$ & $\mathrm{P}$ & $\mathrm{P}$ & $\mathrm{P}$ & $\mathrm{P}$ & $\mathrm{P}$ & $\mathrm{P}$ & $\mathrm{P}$ & $\mathrm{P}$ & $\mathrm{N}$ \\
\hline 6 & $\mathrm{P}$ & $\mathrm{P}$ & $\mathrm{P}$ & $P$ & $\mathrm{P}$ & $\mathrm{P}$ & $\mathrm{P}$ & P & $P$ & $\mathrm{P}$ & $\mathrm{P}$ & $\mathrm{P}$ & $\mathrm{P}$ & $\mathrm{P}$ & $\mathrm{N}$ \\
\hline 7 & $\mathrm{P}$ & $P$ & $\mathrm{P}$ & $\mathrm{P}$ & $\mathrm{P}$ & $\mathrm{P}$ & $\mathrm{P}$ & $\mathrm{P}$ & $P$ & $\mathrm{P}$ & $\mathrm{P}$ & $\mathrm{P}$ & $\mathrm{P}$ & $\mathrm{P}$ & $\mathrm{N}$ \\
\hline 8 & $\mathrm{P}$ & $\mathrm{P}$ & $\mathrm{N}$ & $\mathrm{P}$ & $\mathrm{P}$ & $\mathrm{P}$ & $\mathrm{P}$ & $\mathrm{P}$ & $\mathrm{P}$ & $\mathrm{P}$ & $\mathrm{P}$ & $\mathrm{P}$ & $\mathrm{P}$ & $\mathrm{P}$ & $\mathrm{N}$ \\
\hline 9 & $\mathrm{P}$ & $P$ & $\mathrm{~N}$ & $P$ & $\mathrm{P}$ & $\mathrm{N}$ & $\mathrm{P}$ & $P$ & $P$ & $P$ & $\mathrm{P}$ & $P$ & $\mathrm{P}$ & $\mathrm{N}$ & $\mathrm{N}$ \\
\hline 10 & $\mathrm{P}$ & $\mathbf{P}$ & $\mathrm{N}$ & $P$ & $\mathrm{P}$ & $\mathrm{N}$ & $\mathrm{P}$ & $\mathbf{P}$ & $\mathrm{N}$ & $\mathbf{P}$ & $\mathrm{P}$ & $\mathbf{P}$ & $\mathbf{P}$ & $\mathrm{N}$ & $\mathrm{N}$ \\
\hline 11 & $P$ & $\mathrm{P}$ & $\mathrm{N}$ & $P$ & $\mathrm{P}$ & $\mathrm{P}$ & $\mathrm{P}$ & P & $P$ & $P$ & $\mathrm{P}$ & $P$ & P & $\mathrm{P}$ & $\mathrm{N}$ \\
\hline 12 & $\mathrm{P}$ & $\mathrm{P}$ & $\mathrm{N}$ & $\mathrm{P}$ & $\mathrm{P}$ & $\mathrm{P}$ & $P$ & $\mathrm{P}$ & $\mathrm{P}$ & $\mathrm{P}$ & $\mathrm{P}$ & $P$ & $\mathrm{P}$ & $\mathrm{P}$ & $\mathrm{N}$ \\
\hline 13 & $\mathrm{P}$ & $\mathrm{P}$ & $\mathrm{N}$ & P & $\mathrm{P}$ & $\mathrm{N}$ & $\mathrm{P}$ & $\mathrm{P}$ & $\mathrm{N}$ & $\mathrm{P}$ & $\mathrm{P}$ & P & $\mathrm{P}$ & $\mathrm{P}$ & $\mathrm{N}$ \\
\hline 14 & $\mathrm{P}$ & $P$ & $\mathrm{~N}$ & $\mathrm{P}$ & $\mathrm{N}$ & $\mathrm{N}$ & $\mathrm{N}$ & $\mathrm{N}$ & $\mathrm{N}$ & $P$ & $\mathrm{~N}$ & $\mathrm{~N}$ & $\mathrm{~N}$ & $\mathrm{~N}$ & $\mathrm{~N}$ \\
\hline 15 & $P$ & $P$ & $\mathrm{~N}$ & $P$ & $\mathrm{~N}$ & $\mathrm{~N}$ & $\mathrm{~N}$ & $\mathrm{~N}$ & $\mathrm{~N}$ & $P$ & $\mathrm{~N}$ & $\mathrm{~N}$ & $\mathrm{~N}$ & $\mathrm{~N}$ & $\mathrm{~N}$ \\
\hline 16 & $\mathrm{P}$ & $\mathrm{N}$ & $\mathrm{N}$ & $\mathrm{P}$ & $\mathrm{N}$ & $\mathrm{N}$ & $\mathrm{N}$ & $\mathrm{N}$ & $\mathrm{N}$ & $\mathrm{P}$ & $\mathrm{N}$ & $\mathrm{N}$ & $\mathrm{N}$ & $\mathrm{N}$ & $\mathrm{N}$ \\
\hline 17 & $\mathrm{P}$ & $\mathrm{N}$ & $\mathbf{N}$ & $\mathbf{P}$ & $\mathrm{N}$ & $\mathrm{N}$ & $\mathrm{N}$ & $\mathrm{N}$ & $\mathrm{N}$ & $\mathrm{P}$ & $\mathrm{N}$ & $\mathrm{N}$ & $\mathrm{N}$ & $\mathrm{N}$ & $\mathrm{N}$ \\
\hline 18 & $\mathrm{P}$ & $\mathrm{N}$ & $\mathrm{N}$ & $\mathrm{P}$ & $\mathrm{N}$ & $\mathrm{N}$ & $\mathrm{N}$ & $\mathrm{N}$ & $\mathrm{N}$ & $\mathrm{P}$ & $\mathrm{N}$ & $\mathrm{N}$ & $\mathrm{N}$ & $\mathrm{N}$ & $\mathrm{N}$ \\
\hline 19 & $P$ & $\mathrm{~N}$ & $\mathrm{~N}$ & P & $\mathrm{N}$ & $\mathrm{N}$ & $\mathrm{N}$ & $\mathrm{N}$ & $\mathrm{N}$ & $P$ & $\mathrm{~N}$ & $\mathrm{~N}$ & $\mathrm{~N}$ & $\mathrm{~N}$ & $\mathrm{~N}$ \\
\hline 20 & $\mathrm{P}$ & $\mathrm{N}$ & $\mathrm{N}$ & $\mathrm{P}$ & $\mathrm{N}$ & $\mathrm{N}$ & $\mathrm{N}$ & $\mathrm{N}$ & $\mathrm{N}$ & $\mathrm{P}$ & $\mathrm{N}$ & $\mathrm{N}$ & $\mathrm{N}$ & $\mathrm{N}$ & $\mathrm{N}$ \\
\hline 21 & $\mathrm{~N}$ & $\mathrm{~N}$ & $\mathrm{~N}$ & $\mathrm{~N}$ & $\mathrm{~N}$ & $\mathrm{~N}$ & $\mathrm{~N}$ & $\mathrm{~N}$ & $\mathrm{~N}$ & $\mathrm{P}$ & $\mathrm{N}$ & $\mathrm{N}$ & $\mathrm{N}$ & $\mathrm{N}$ & $\mathrm{N}$ \\
\hline 22 & $\mathrm{~N}$ & $\mathrm{~N}$ & $\mathrm{~N}$ & $\mathrm{~N}$ & $\mathrm{~N}$ & $\mathrm{~N}$ & $\mathrm{~N}$ & $\mathrm{~N}$ & $\mathrm{~N}$ & $\mathrm{~N}$ & $\mathrm{~N}$ & $\mathrm{~N}$ & $\mathrm{~N}$ & $\mathrm{~N}$ & $\mathrm{~N}$ \\
\hline 23 & $\mathrm{~N}$ & $\mathrm{~N}$ & $\mathrm{~N}$ & $\mathrm{~N}$ & $\mathrm{~N}$ & $\mathrm{~N}$ & $\mathrm{~N}$ & $\mathrm{~N}$ & $\mathrm{~N}$ & $\mathrm{~N}$ & $\mathrm{~N}$ & $\mathrm{~N}$ & $\mathrm{~N}$ & $\mathrm{~N}$ & $\mathrm{~N}$ \\
\hline 24 & $\mathrm{~N}$ & $\mathrm{~N}$ & $\mathrm{~N}$ & $\mathrm{~N}$ & $\mathrm{~N}$ & $\mathrm{~N}$ & $\mathrm{~N}$ & $\mathrm{~N}$ & $\mathrm{~N}$ & $\mathrm{~N}$ & $\mathrm{~N}$ & $\mathrm{~N}$ & $\mathrm{~N}$ & $\mathrm{~N}$ & $\mathrm{~N}$ \\
\hline
\end{tabular}

oscilações foram observadas, em especial na diluição 1:200, com animais com resultados negativos por uma ou duas semanas, positivando novamente na terceira. Entretanto os animais só foram considerados negativos, para cada um dos títulos, quando se apresentavam negativos por três semanas consecutivas. $\mathrm{Na}$ diluição 1:50, o bezerro 4 manteve-se positivo por 21 semanas; os bezerros 1 e 2 por 20 semanas e os outros dois por 13 semanas. $\mathrm{Na}$ diluição 1:100, o bezerro 1 manteve-se positivo por 15 semanas e os outros quatro por 13 semanas. $\mathrm{Na}$ diluição 1:200, o bezerro 5 apresentou títulos de anticorpos por uma semana e os bezerros 1, 2, 3 e 4 por 7, 12, 12 e 13 semanas, respectivamente.

Segundo Tizard ${ }^{43}$, a falha da transferência de anticorpos maternais através do colostro pode ocorrer por produção de colostro insuficiente ou de má qualidade pela mãe; falha de ingestão por parte do animal recém-nascido e/ou falha de absorção dos anticorpos através do epitélio intestinal, fatos que podem ter ocorrido neste estudo.

Em ruminantes, a placenta não permite a transferência de imunoglobulina materna para a circulação fetal, por isso qualquer anticorpo no soro pré-colostral é derivado do feto que indica uma resposta imune ativa contra a invasão do parasito. Durante as primeiras 24-36 horas de vida o bezerro é hábil pra absorver imunoglobulinas do leite da mãe através do intestino e, conseqüentemente, os anticorpos têm especificidade idêntica da mãe $e^{44}$. Hietala e Thurmond ${ }^{28}$ e Innes et al. ${ }^{45}$ usando bezerros soronegativos pré-colostral nascidos de vacas soropositivas que soroconverteram após a ingestão de colostro demonstraram que a resposta de anticorpos anti- $N$. caninum declinou ao redor de 19,6 dias e 21 dias após o nascimento, respectivamente.

Hietala e Thurmond ${ }^{28}$ relataram que bezerros nascidos negativos e que receberam colostro de vacas $N$. caninum positivas, tornaram-se soropositivos e os anticorpos colostrais declinaram aos 128 dias após o nascimento, isto é ao redor da $18^{\mathrm{a}}$ semana após o nascimento. Estes resultados foram semelhantes aos do presente estudo, no qual na semana 21 foi observado o desaparecimento dos anticorpos colostrais 
em todos os bezerros na menor diluição utilizada na RIFI.

No trabalho realizado por Hietala e Thurmond ${ }^{28}, 17$ dos 284 bezerros classificados como negativos para $N$. caninum soroconverteram e 13 destas soroconversões ocorreram no período neonatal seguido da ingestão de colostro, no qual as mães de nove bezerros eram soropositivos, uma soronegativa e três não tinham o diagnóstico. Assim, foi sugerido que os bezerros que soroconverteram podem ter sido infectados durante os primeiros meses de vida; infectados congenitamente, mas não tiveram uma resposta de anticorpos detectáveis no nascimento e a sensibilidade e especificidade do teste empregado pode ter influenciado no diagnóstico da soroconversão, como também mostrado por Davison, Guy e Otter ${ }^{17}$.

\section{Conclusões}

Estes resultados mostram que bezerros recém-nascidos soronegativos précolostrais quando alimentados com colostro de diferentes vacas podem apresentar resultados sorológicos positivos para $N$. caninum devido à absorção de anticorpos colostrais. Este encontro em bezerros com até quatro meses de idade deve ser sempre interpretado com grande cautela, em especial em animais alimentados com pool de colostro, nos quais não se pode identificar com exatidão as vacas doadoras. Exames pareados bezerros e suas mães são importantes para melhor avaliação dos resultados da sorologia, entretanto nas propriedades que utilizam pool de colostro, a avaliação sorológica dos bezerros só deve ser realizada em animais com idade mínima de quatro meses.

\section{Serological profile of colostral antibodies to Neospora caninum in infection free calves}

\begin{abstract}
Neospora caninum is considered the main cause of bovine abortion worldwide. The correct laboratorial diagnose is very important to identify the infected animals and to apply control measure. The objetive of this study was to show the persistence period of colostral antibodies in calves. Eight newborn Holstein Friesan calves, males, were selected from N. caninum soronegative dams. Pre-colostral blood samples were collected of these calves and all of them were seronegative to $N$. caninum. They were fed with two liters of pooled colostrum from seropositive cows within two hours after birth. Blood samples were collected and tested weekly until the animals turned negative. Serum samples were tested for antibodies to $\mathrm{N}$. caninum using indirect fluorescence antibody test at 1:50; 1: 100 and 1:200 dilutions. Antibodies were not detected from three out of eight calves and they were excluded from the study. The remaining 5 calves seroconverted in all dilutions at the fifth day after colostrums ingestion. At 1:50 dilution, one calf remained positive for 21 weeks, two for 20 weeks and one for 13 weeks. At 1:100, one calf was positive for 15 weeks and the remaining 4 calves for 13 weeks. At 1:200, each calf was positive for 1, 7,12,12 and 13 weeks, respectively. These results demonstrate that the colostral antibody to $N$. caninum may persist until 21 weeks after birth in calves and it's very important to exclud the calves at the first month of age in the seroprevalence studies to avoid the false-positive results.
\end{abstract}

Key words: Neospora. Bovine. Calves. Colostrum. Serology. 


\section{Referências}

1 BJERKAS, I.; MOHN, S. F.; PRESTHUS, J. Unidentified cyst-forming sporozoon causing encephalomyelitis and myositis in dogs. Zeithschrifft Fuer Parasitenkunde, v. 70, p. 271-274, 1984.

2 DUBEY, J. P.; CARPENTER, J. L.; SPEER, C. A.; TOPPER, M. J.; UGGLA, A. Newly recognized fatal protozoan disease of dogs. Journal of the American Veterinary Medical Association, v. 192, p. 1269-1285, 1988.

3 DUBEY, J. P. Review of Neospora caninum and neosporosis in animals. Korean Journal of Parasitology, v. 41, p. 1-16, 2003.

4 MCALLISTER, M. M.; DUBEY, J. P.; LINDSAY, D. S.; JOLLEY, W. R.; WILLS, R. A.; MCGUIRE, A. M. Dogs are definitive hosts of Neospora caninum. International Journal for Parasitology, v. 28, p. 1473-1478, 1998.

5 LINDSAY, D. S.; DUBEY, J. P.; DUNCAN, R. B. Confirmation that the dog is a definitive host for Neospora caninum. Veterinary Parasitology, v. 82, p. 327-333, 1999.

6 LINDSAY, D. S.; UPTON, S. J.; DUBEY, J. P. A structural study of the Neospora caninum oocysts. International Journal for Parasitology, v. 29, p. 15211523, 1999 .

7 GONDIM, L. P.; MCALLISTER, M. M.; PITT, W. C.; ZEMLICKA, D. E. Coyotes (Canis latrans) are definitive hosts of Neospora caninum. International Journal for Parasitology, v. 34, p. 159-161, 2004.

8 PARÉ, J.; THURMOND, M. C.; HIETALA, S. K. Congenital Neospora caninum infection in dairy cattle and associated calfhood mortality. Canadian Journal of Veterinary Research, v. 60, p. 133-139, 1996.

9 BJÖRKMAN, C.; JOHANSSON, O.; STENLUND, S.; HOLMDAHL, O. J. M; UGGLA, A. Neospora species infection in a herd of dairy cattle. Journal of the American Veterinary Medical Association, v. 208, p. 1441-1444, 1996.

10 ANDERSON, M. L.; REYNOLDS, J. P.; ROWE, J. D.; SVERLOW, K. W.; PACKHAM, A. E.; BARR, B. C.; CONRAD, P. A. Evidence of vertical transmission of Neospora sp. infection in dairy cattle. Journal of the American Veterinary Medical Association, v. 210, p. 1169-1172, 1997.

11 PARÉ, J.; THURMOND, M. C.; HIETALA, S. K. Neospora caninum antibodies in cows during pregnancy as a predictor of congenital infection and abortion. Journal of Parasitology, v. 83, p. 82-87, 1997.

12 THURMOND, M. C.; HIETALA, S. Effect of congenitally acquired Neospora caninum infection on risk of abortion and subsequent abortions in dairy cattle. American Journa1 of Veterinary Research, v. 58, p. 1381-1385, 1997.

13 SCHARES, G.; PETERS, M.; WURM, R.; BÄRWALD,
A.; CONRATHS, F. J. The efficiency of vertical transmission of Neospora caninum in dairy cattle ana1ysed by serologica 1 techniques. Veterinary Parasitology, v. 80, p. 87-98, 1998.

14 WOUDA, W.; MOEN, A. R.; SCHUKKEN, Y. H. Abortion risk in progeny of cows after a Neospora caninum epidemic. Theriogenology, v. 49, p. 1311 1316, 1998.

15 WOUDA, W.; BARTELS, C. J. M.; MOEN, A. R. Characteristics of Neospora caninum-associated abortion storms in dairy herds in the Netherlands (1995-1997). Theriogenology, v. 52, p. 233-245, 1999.

16 DAVISON, H. C.; GUY, F.; OTTER, A.; TREES, A. J. Significance of Neospora caninum in British dairy cattle determined by estimation of seroprevalence in normally calving cattle and aborting cattle. International Journal for Parasitology, v. 29, p. 1189-1194, 1999.

17 DAVISON, H. C.; GUY, F.; OTTER, A. Estimation of vertical and horizontal transmission parameters of Neospora caninum infections in dairy cattle. International Journal for Parasitology, v. 29, p. 16831689, 1999.

18 JENSEN, A. M.; BJÖRKMAN, C.; KJELDSEN, A. M.; WEDDERKOPP, A.; WILLADSEN, C.; UGGLA, A.; LIND, P. Associations of Neospora caninum seropositivity with gestation number and pregnancy outcome in Danish dairy herds. Preventive Veterinary Medicine, v. 40, p. 151-163, 1999.

19 WALDNER, C. L.; JANZEN, E. D.; HENDERSON, J.; HAINES, D. M. Outbreak of abortion associated with Neospora caninum infection in a beef herd. Journal of the American Veterinary Medical Association, v. 215, p. 1485-1490, 1999.

20 ATKINSON, R. A.; COOK, R. W.; REDDACLIFF, L. A.; ROTHWELL, J.; BROADY, K. W.; HARPER, P. A. W.; ELLIS, J. T. Seroprevalence of Neospora caninum infection following an abortion outbreak in a dairy cattle herd. Australian Veterinary Journal, v. 78, p. 262-266, 2000.

21 BERGERON, N.; fECTEAU, G.; PARÉ, J.; MARTINEAU, R.; VILLENEUVE, A. Vertical and horizontal transmission of Neospora caninum in dairy herds in Québec. Canadian Veterinary Journal, v. 41, p. 464-469, 2000.

22 DIJKSTRA, T.; BARKEMA, H. W.; EYSKER, M.; WOUDA, W. Evidence of post-natal transmission of Neospora caninum in Dutch dairy herds. International Journal for Parasitology, v. 31, p. 209-215, 2001.

23 MCALLISTER, M.; HUFFMAN, E. M.; HIETALA, S. K.; CONRAD, P. A.; ANDERSON, M. L.; SALMAN, $M$. D. Evidence suggesting a point source exposure in an outbreak of bovine abortion due to neosporosis. Journal of Veterinary Diagnostic Investigation, v. 8, p. 355-357, 1996.

24 THURMOND, M. C.; HIETALA, S. K.; BLANCHARD, P. C. Herd-based diagnosis of Neospora 
caninum-induced endemic and epidemic abortion in cows and evidence for congenital and postnatal transmission. Journal of Veterinary Diagnostic Investigation, v. 9, p. 44-49, 1997.

25 COX, B. T.; REICHEL, M. P.; GRIFFITHS, L. M. Serology of a Neospora abortion outbreak on a dairy farm in New Zealand: A case study. New Zealand Veterinary Journal, v. 46, p. 28-31, 1998.

26 WALDNER, C. L.; JANZEN, E. D.; RIBBLE, C. S. Determination of the association between Neospora caninum infection and reproductive performance in beef herds. Journal of the American Veterinary Medical Association, v. 213, p. 685-690, 1998.

27 BJORKMAN, C.; NASLUND, K.; STENLUND, S.; MALEY, S. W.; BUXTON, D.; UGGLA, A. An IgG avidity ELISA to discriminate between recent and chronic Neospora caninum infection. Journal of Veterinary Diagnostic Investigation, v. 11, p. 41-44, 1999.

28 HIETALA, S. K.; THURMOND, M. C. Postnatal Neospora caninum transmission and transient serologic responses in two dairies. International Journal for Parasitology, v. 29, p. 1669-1676, 1999.

29 PATITUCCI, A. N.; CHARLESTON, W. A. G.; ALLEY, M. R. et al. Serological study of a dairy herd with a recent history of Neospora abortion. New Zealand Veterinary Journal, v. 47, p. 28-30, 1999.

30 SCHARES, G.; CONRATHS, F. J.; REICHEL, M. P. Bovine neosporosis: Comparasion of serological methods using outbreak sera from a dairy herd in New Zealand. International Journal for Parasitology, v. 29, p. 1659-1667, 1999.

31 DYER, R. M.; JENKJNS, M. C.; KWOK, O. C. H.; DOUGLAS, L. W.; DUBEY, J. P. Serologic survey of Neospora caninum infection in a closed dairy cattle herd in Maryland: Risk of serologic reactivity by production groups. Veterinary Parasitology, v. 90, p. 171-181, 2000.

32 JENKINS, M. C.; CAVER, J. A.; BJÖRKMAN, C.; ANDERSON, T. A.; ROMAND, S.; VINYARD, B.; UGGLA, A.; THULLIEZ, P.; DUBEY, J. P. Serological investigation of an outbreak of Neospora caninumassociated abortion in a dairy herd in southeastern United States. Veterinary Parasitology, v. 94, p. 17-26, 2000.

33 MCALLISTER, M. M.; BJÖRKMAN, C.; ANOERSON-SPRECHER, R.; ROGERS, D. G. Evidence of point-source exposure to Neospora caninum and protective immunity in a herd of beef cows. Journal of the American Veterinary Medical Association, v. 217, p. $881-887,2000$.

34 DIJKSTRA, T.; EYSKER, M.; SCHARES, G.; CONRATHS, F. J.; WOUDA, W.; BARKEMA, H. W. Dogs shed Neospora caninum oocysts after ingestion of naturally infected bovine placenta but not after ingestion of colostrum spiked with Neospora caninum tachyzoites. International Journal for Parasitology, v. 31, p. 747-752, 2001.

35 DIJKSTRA, T.; BARKEMA, H. W.; EYSKER, M.; HESSELINK, J. W.; WOUDA, W. Natural transmission routes of Neospora caninum between farm dogs and cattle. Veterinary Parasitology, v. 105, p. 99-104, 2002.

36 SCHARES, G.; BÄRWALD, A.; STAUBACH, C.; SÖNDGEN, P.; RAUSER, M.; SCHRÖDER, R.; PETERS, M.; WURM, R.; SELHORST, T.; CONRATHS, F.J. p38avidity-ELISA: Examination of herds experiencing epidemic or endemic Neospora caninum-associated bovine abortion. Veterinary Parasitology, v. 106, p. 293-305, 2002.

37 STENLUND, S.; KINDAHL, H.: UGGLA, A.; BJÖRKMAN, C. A long-term study of Neospora caninum infection in a Swedish Dairy Herd, Acta Veterinary Scandinavica, v. 44, p. 63-71, 2003.

38 KASHIWAZAKI, Y.; GIANNEECCHINI, R. E.; LUST, M.; GIL, J. Seroepidemiology of neosporosis in dairy cattle in Uruguay, Veterinary Parasitology, v. 120, p. 139-144, 2004.

39 COLE, R. A.; LINDSAY, D. S.; BLAGBURN, B. L.; DUBEY, J. P. Vertical transmission of Neospora in mice. Journal for Parasitology, v. 81, p. 730-732, 1995.

40 UGGLA, A.; STENLUND, S.; HOLMDAHL, O. J. M.; JAKUBEK, E. B.; THEBO, P.; KINDAHL, H.; BJÖRKMAN, C. Oral Neospora caninum inoculation of neonatal calves. Internationa1 Journa1 for Parasitology, v. 28, p. 1467-1472, 1998.

41 DAVISON, H. C.; GUY, C. S.; MCGARRY, J. W.; GUY, E.; WILLIAMS, D. J. L.; TREES, A. J. Experimental studies on the transmission of Neospora caninum between cattle. Research in Veterinary Science, v. 70, p. 163-168, 2001.

42 DUBEY, J. P.; HATTEL, A. L; LINDSAY, D. S.; TOPPER, M. J. Neonatal Neospora caninum infection in dogs: Isolation of the causative agent experimental transmission. Journal of the American Veterinary Medical Association, v. 193, n. 10, p. 1259-1263, 1988.

43 TIZARD, I. R. Imunologia veterinária: uma introdução. In: TIZARD, I. R. Imunidade no feto e no recém-nascido. São Paulo: Roca, 2002. p. 233-246.

44 STOTT, G. H.; MARX, D. B.; MENEFEE, B. E.; NIGHTENGALE, G. T. Colostral immunoglobulin transfer in calves. I. Period of absorption. Journal Dairy Science, v. 62, p. 1632-1638, 1979.

45 INNES, E. A; WRIGHT, S. E.; MALEY, S.; RAE, A.; SCHOCK, A.; KIRVAR, E.; BARTLEY, P.; HAMILTON, C.; CAREY, I. M.; BUXTON, D. Protection against vertical transmission in bovine neosporosis. International Journal for Parasitology, v. 31, p. 15231534, 2001. 